\title{
Primal-Dual Bilinear Programming Solution of the Absolute Value Equation
}

\author{
Olvi L. Mangasarian*
}

\begin{abstract}
We propose a finitely terminating primal-dual bilinear programming algorithm for the solution of the NP-hard absolute value equation (AVE): $A x-|x|=b$, where $A$ is an $n \times n$ square matrix. The algorithm, which makes no assumptions on AVE other than solvability, consists of a finite number of linear programs terminating at a solution of the AVE or at a stationary point of the bilinear program. The proposed algorithm was tested on 500 consecutively generated random instances of the AVE with $n=10,50,100,500$ and 1,000. The algorithm solved $88.6 \%$ of the test problems to an accuracy of $1 e-6$.
\end{abstract}

Keywords: absolute value equation, bilinear programming, linear programming

\section{INTRODUCTION}

We consider the absolute value equation (AVE):

$$
A x-|x|=b,
$$

where $A \in R^{n \times n}$ and $b \in R^{n}$ are given, and $|\cdot|$ denotes absolute value. A slightly more general form of the AVE, $A x+B|x|=b$ was introduced in [14] and investigated in a more general context in [9]. The AVE (1.1) was investigated in detail theoretically in [11], and a bilinear program in the primal space of the problem was prescribed there for the special case when the singular values of $A$ are not less than one. No computational results were given in either [11] or [9]. In contrast in [8], computational results were given for a linear-programming-based successive linearization algorithm utilizing a concave minimization model. As was shown in [11], the general NP-hard linear complementarity problem (LCP) $[3,4,2]$, which subsumes many mathematical programming problems, can be formulated as an AVE (1.1). This implies that (1.1) is NP-hard in its general form. More recently a generalized Newton method was proposed for solving the AVE [10], while a uniqueness result for the AVE is presented in [15] and for a more general version of the AVE in [16], and finally existence and convexity results are given in $[6]$.

Our point of departure here is to look at the AVE in its primal and dual spaces of the problem and formulate an algorithm that minimizes a bilinear function (that is the scalar product of two linear functions) in the combined primal-dual space which has a global minimum of zero that yields an exact solution of the AVE. In Section 2 we describe our bilinear formulation of the AVE and show that a zero minimum of the bilinear program yields a solution to the AVE. In Section 3 of the paper we state our algorithm for the bilinear program consisting of a succession of linear programs that terminate at a global solution of the the AVE or at a stationary point of the bilinear program. In Section 4 we give computational results that show the effectiveness of our approach by solving $88.6 \%$ of a sequence of 500

\footnotetext{
${ }^{*}$ Computer Sciences Department, University of Wisconsin, Madison, WI 53706 and Department of Mathematics, University of California at San Diego, La Jolla, CA 92093.olvi@cs.wisc.edu.
} 
randomly generated consecutive AVEs in $R^{10}$ to $R^{1,000}$ to an accuracy of $1 e-6$. Section 5 concludes the paper.

We describe our notation now. All vectors will be column vectors unless transposed to a row vector by a prime'. For a vector $x \in R^{n}$ the notation $x_{j}$ will signify the $j$-th component. The scalar (inner) product of two vectors $x$ and $y$ in the $n$-dimensional real space $R^{n}$ will be denoted by $x^{\prime} y$. For $x \in R^{n}$,

$\|x\|$ denotes the 2-norm: $\left(\sum_{i=1}^{n}\left(x_{i}\right)^{2}\right)^{\frac{1}{2}}$. The notation $A \in R^{m \times n}$ will signify a real $m \times n$ matrix. For such a matrix, $A^{\prime}$ will denote the transpose of $A$. A vector of ones in a real space of arbitrary dimension will be denoted by $e$. Thus for $e \in R^{m}$ and $y \in R^{m}$ the notation $e^{\prime} y$ will denote the sum of the components of $y$. A vector of zeros in a real space of arbitrary dimension will be denoted by 0 . The abbreviation "s.t." stands for "subject to".

\section{Bilinear Formulation of the Absolute Value Equation}

We begin with the linear program:

$$
\min _{x, y} h^{\prime} y \text { s.t. } A x-y=b, x+y \geq 0,-x+y \geq 0,
$$

and its dual:

$$
\max _{u, v, w} b^{\prime} u \text { s.t. } A^{\prime} u+v-w=0,-u+v+w=h,(v, w) \geq 0,
$$

where $h$ is some vector in $R^{n}$ that will play a key role in a bilinear programming formulation. We now state the following simple lemma.

LEMma 2.1. Let $(x, y)$ be a solution of the primal problem (2.2) and $(u, v, w)$ be a solution of the corresponding dual problem (2.3). Then:

$$
v+w>0 \Longrightarrow A x-|x|=b
$$

Proof From the complementarity condition we have that:

$$
v^{\prime}(x+y)+w^{\prime}(-x+y)=0 .
$$

Hence, if $v+w>0$ it follows that either $(x+y)_{i}=0$, or $(-x+y)_{i}=0$, for $i=1, \ldots, n$. Hence $y=|x|$ and from the constraint $A x-y=b$ it follows that $A x-|x|=b$.

Based on this lemma it follows that for a primal-dual optimal solution $(x, y, u, v, w)$, if $v+w \geq \epsilon e$ for a positive $\epsilon$, then $A x-|x|=b$. Furthermore, from the dual constraints we have that $h=-u+v+w$ and hence the difference between the primal and dual objective functions evaluated at a primal-dual feasible point becomes:

$$
h^{\prime} y-b^{\prime} u=(-u+v+w)^{\prime} y-b^{\prime} u \geq 0,
$$

where the inequality of (2.6) follows from the fact that at a primal-dual feasible point, the primal objective function exceeds or equals the dual objective function. At a primal-dual optimal point this difference is zero. Hence combining these statements with Lemma 2.1 and the extra imposed condition that $v+w \geq \epsilon e$, we have the following proposition. 
Proposition 2.2. Equivalence of AVE and Zero Minimum of the Bilinear Program At a zero minimum of the following bilinear program:

$$
\begin{aligned}
\min _{x, y, u, v, w} y^{\prime}(-u+v+w)-b^{\prime} u & \\
\text { s.t. } & =b \\
x-y & \geq 0 \\
-x+y & \geq 0 \\
A^{\prime} u+v-w & =0 \\
v+w & \geq \epsilon e \\
(v, w) & \geq 0
\end{aligned}
$$

we have that $y=|x|$ and $A x-|x|=b$ for any solution point $(x, y, u, v, w)$.

We establish now the existence of a zero-minimum solution to the bilinear program (2.7) under the assumption that AVE (1.1) is solvable.

Proposition 2.3. Existence of a Zero-Minimum Solution to the Bilinear Program Under the assumption that the absolute value equation (1.1) is solvable, the bilinear program (2.7) has a zero minimum solution $(x, y, u, v, w)$ such that $x$ solves the absolute value equation (1.1).

Proof Since AVE (1.1) has a solution, say $x$, then the feasible region of the bilinear program (2.7) is nonempty because the point $(x, y=|x|, u=0, v=w=\epsilon e / 2)$ satisfies the constraints of (2.7). Hence the quadratic bilinear objective function of (2.7) which by Proposition 2.2 is bounded below by zero must by [5] have a solution. Since by Proposition 2.2 a zero-minimum solution solves AVE, and AVE is solvable by assumption, such a zero-minimum solution exists that solves AVE.

We now present a computational algorithm for solving the bilinear program (2.7) that consists of solving a finite number of linear programs.

\section{Bilinear Programming Algorithm for the Absolute Value Equation}

We begin by stating our bilinear algorithm as follows.

Algorithm 3.1. Choose parameter value $\epsilon$ for the constraint of (2.7) (typically $\epsilon=1 e-2$ ), tolerance (typically tol=1e-6), and maximum number of iterations itmax (typically itmax=40).

(I) Initialize the algorithm by determining an initial $\left(x^{0}, y^{0}\right)$ by solving the following linear program:

$$
\begin{array}{lrl}
\min _{x, y} & e^{\prime} y \\
\text { s.t. } & A x-y=b \\
& x+y \geq 0 \\
& -x+y \geq 0
\end{array}
$$

Set iteration number $i=0$.

(II) While $\left\|A x^{i}-\left|x^{i}\right|-b\right\|>t o l$, the bilinear objective function of (2.7) is decreasing, and $i \leq i$ max perform the following three steps.

(III) Solve the following linear program for $\left(u^{i+1}, v^{i+1}, w^{i+1}\right)$ :

$$
\begin{array}{ll}
\min _{u, v, w} y^{i^{\prime}}(-u+v+w)-b^{\prime} u & \\
\text { s.t. } & A^{\prime} u+v-w=0 \\
v+w & \geq \epsilon e \\
(v, w) & \geq 0
\end{array}
$$


(IV) Solve the following linear program for $\left(x^{i+1}, y^{i+1}\right)$ :

$$
\begin{array}{rlrl}
\min _{x, y} & \left(-u^{i+1}+v^{i+1}+w^{i+1}\right)^{\prime} y & \\
\text { s.t. } & A x-y=b \\
x+y & \geq 0 \\
-x+y & \geq 0
\end{array}
$$

(V) $i=i+1$. Go to Step (II).

We establish now finite termination of our bilinear algorithm.

Proposition 3.2. Finite Termination of the Bilinear Algorithm Under the assumption that the absolute value equation (1.1) is solvable and the maximum number of iterations itmax is sufficiently large, the Bilinear Algorithm 3.1 terminates in a finite number of iterations at a global zero-minimum point that solves the absolute value equation (1.1), or at iteration $i$ with a solution $\left(x^{i+1}, y^{i+1}, u^{i+1}, v^{i+1}, w^{i+1}\right)$ that satisfies the following minimum principle necessary optimality condition for the bilinear program (2.7):

$$
\begin{gathered}
\left(-u^{i+1}+v^{i+1}+w^{i+1}\right)^{\prime}\left(y-y^{i+1}\right)-\left(y^{i+1}+b\right)^{\prime}\left(u-u^{i+1}\right)+y^{i+1^{\prime}}\left(v-v^{i+1}\right)+y^{i+1^{\prime}}\left(w-w^{i+1}\right) \geq 0, \\
\forall x \in X,(u, v, w) \in U,
\end{gathered}
$$

where

$$
\begin{gathered}
X=\{(x, y) \mid A x-y=b, x+y \geq 0,-x+y \geq 0\}, \\
U=\left\{(u, v, w) \mid A^{\prime} u+v-w=0, v+w \geq \epsilon e,(v, w) \geq 0\right\} .
\end{gathered}
$$

Proof Note first that the sets $X$ and $U$ defined above are nonempty because as pointed out earlier that under the assumption that AVE has a solution $x$ then $(x,|x|) \in X$ and $(0, \epsilon e / 2, \epsilon e / 2) \in U$. To keep the proof simple we shall assume that neither $X$ nor $U$ have straight lines going infinity in both directions. This assumption which allows us to utilize [13, Corollary 32.3.4], can be easily achieved by defining $x=x_{I}-x_{I I}, x_{I} \geq 0, x_{I I} \geq 0$ and $u=u_{I}-u_{I I}, u_{I} \geq 0, u_{I I} \geq 0$. Hence, the bilinear program (2.7) with an objective function bounded below by zero, which is equivalent to a concave function minimization [1, Proposition 2.2], has a vertex solution on the polyhedral set $X \times U$. If for some $i$ th iteration the bilinear objective function does not decrease, then each of the linear programs of steps (III) and (IV) of the algorithm must have returned $\left(x^{i+1}, y^{i+1}\right)$ and $\left(u^{i+1}, v^{i+1}, w^{i+1}\right)$ such that:

$y^{i+1^{\prime}}(-u+v+w)-b^{\prime} u \geq y^{i+1^{\prime}}\left(-u^{i}+v^{i}+w^{i}\right)-b u^{i}=y^{i+1^{\prime}}\left(-u^{i+1}+v^{i+1}+w^{i+1}\right)-b u^{i+1}, \forall(u, v, w) \in U$,

and

$$
\left(-u^{i+1}+v^{i+1}+w^{i+1}\right)^{\prime} y \geq\left(-u^{i+1}+v^{i+1}+w^{i+1}\right)^{\prime} y^{i}=\left(-u^{i+1}+v^{i+1}+w^{i+1}\right)^{\prime} y^{i+1}, \forall(x, y) \in X .
$$

Combining the inequalities of (3.14) and (3.15) gives the minimum principle necessary optimality condition (3.11). Since there are a finite number of vertices of the set $X \times U$, and since each vertex visited by Algorithm 3.1 gives a lesser value for the bilinear objective than the previous vertex, no vertex is repeated. Thus our algorithm must terminate at either a global zero minimum solution or a point satisfying the minimum principle necessary optimality condition.

We turn now to our computational results. 


\section{Computational Results}

We implemented our algorithm by solving 500 solvable random instances of the absolute value equation (1.1) consecutively generated. Elements of the matrix $A$ were random numbers picked from a uniform distribution in the interval $[-5,5]$. A random solution $x$ with random components from $[-.5, .5]$ was generated and the right hand side $b$ was computed as $b=A x-|x|$. All computation was performed on 4 Gigabyte machine running i386 rhe15 Linux. We utilized the CPLEX linear programming code [7] within MATLAB [12] to solve our linear programs.

Of the 500 test problems, $88.6 \%$ were solved exactly to a tolerance set to $t o l=1 e-6$. The maximum number of iterations was set at 40 . The computational results are summarized in Table 1.

\begin{tabular}{|c|c|c|}
\hline $\begin{array}{c}\text { Problem Size } \\
\mathrm{n}\end{array}$ & $\begin{array}{c}\text { Number of AVEs out of 100 } \\
\text { with 2-norm error } \leq \text { tol=1e-6 }\end{array}$ & $\begin{array}{c}\text { Time in Seconds for } \\
\text { Solving 100 Equations }\end{array}$ \\
\hline 10 & 90 & 1.805 \\
\hline 50 & 87 & 5.725 \\
\hline 100 & 88 & 20.605 \\
\hline 500 & 88 & $1,996.6$ \\
\hline 1,000 & 90 & 19,008 \\
\hline
\end{tabular}

Table 1: Computational Results for 500 Randomly Generated Consecutive AVEs

\section{Conclusion and Outlook}

We have proposed a bilinear programming formulation for solving the NP-hard absolute value equation. The bilinear program was solved by a finite succession of linear programs. In $88.6 \%$ of 500 instances, for each solvable random test problem, the proposed algorithm solved the problem to an accuracy of $1 e-6$. Possible future work may consist of precise sufficient conditions under which the proposed formulation and solution method is guaranteed to solve this NP-hard problem exactly.

Acknowledgments The research described in this Data Mining Institute Report 11-01, February 2011, was supported by the Microsoft Corporation and ExxonMobil.

\section{References}

[1] K. P. Bennett and O. L. Mangasarian. Bilinear separation of two sets in n-space. Computational Optimization and Applications, 2:207-227, 1993.

[2] S.-J. Chung. NP-completeness of the linear complementarity problem. Journal of Optimization Theory and Applications, 60:393-399, 1989.

[3] R. W. Cottle and G. Dantzig. Complementary pivot theory of mathematical programming. Linear Algebra and its Applications, 1:103-125, 1968.

[4] R. W. Cottle, J.-S. Pang, and R. E. Stone. The Linear Complementarity Problem. Academic Press, New York, 1992.

[5] M. Frank and P. Wolfe. An algorithm for quadratic programming. Naval Research Logistics Quarterly, 3:95-110, 1956.

[6] Sheng-Long Hu and Zheng-Hai Huang. A note on absolute equations. Optimization Letters, 4:417-424, 2010.

[7] ILOG, Incline Village, Nevada. ILOG CPLEX 9.0 User's Manual, 2003. http://www.ilog.com/products/cplex/. 
[8] O. L. Mangasarian. Absolute value equation solution via concave minimization. Optimization Letters, 1(1):3-8, 2007. ftp://ftp.cs.wisc.edu/pub/dmi/tech-reports/06-02.pdf.

[9] O. L. Mangasarian. Absolute value programming. Computational Optimization and Applications, 36(1):4353, 2007. ftp://ftp.cs.wisc.edu/pub/dmi/tech-reports/05-04.ps.

[10] O. L. Mangasarian. A generlaized newton method for absolute value equations. Technical Report 08-01, Data Mining Institute, Computer Sciences Department, University of Wisconsin, May 2008. ftp://ftp.cs.wisc.edu/pub/dmi/tech-reports/08-01.pdf. Optimization Letters 3(1), January 2009, 101-108. Online version: http://www.springerlink.com/content/c076875254r7tn38/.

[11] O. L. Mangasarian and R. R. Meyer. Absolute value equations. Linear Algebra and Its Applications, 419:359-367, 2006. ftp://ftp.cs.wisc.edu/pub/dmi/tech-reports/05-06.pdf.

[12] MATLAB. User's Guide. The MathWorks, Inc., Natick, MA 01760, 1994-2006. http://www.mathworks.com.

[13] R. T. Rockafellar. Convex Analysis. Princeton University Press, Princeton, New Jersey, 1970.

[14] J. Rohn. A theorem of the alternatives for the equation $A x+B|x|=b$. Linear and Multilinear Algebra, 52(6):421-426, 2004. http://www.cs.cas.cz/ rohn/publist/alternatives.pdf.

[15] J. Rohn. On unique solvability of the absolute value equation. Optimization Leters, 3:603-606, 2009.

[16] J. Rohn. A residual existence theorem for linear equations. Optimization Letters, 4:287-292, 2010. 